\title{
JAXA Research and Development Report
}

\section{Development of a Plasma Armature Railgun with Two Distributed Power Supplies}

Akira Yamori

March 2004 


\title{
Development of a Plasma Armature Railgun with Two Distributed Power Supplies
}

\author{
By
}

Akira YAMORI

\begin{abstract}
We have been developing a plasma armature railgun with two distributed power supplies as the first step to produce a railgun with multiple distributed power supplies, which is considered as a promising tool for hypervelocities. The railgun has almost the same system and operational principle as those of the twostage plasma armature railgun, which had been developed in our laboratory, except that the two power supplies are distributed along segmented rails or continuous rails. To date, we have performed 11 shots to study the railgun. For the operation of the railgun with two distributed power supplies, to control the generation of a compact plasma armature in the $2^{\text {nd }}$ railgun bore is the most important. In 8 shots the compact structure of the plasma armature was not generated due to a large amount of leakage of the plasma armature around the joint between the two railguns. However in the remaining 3 shots, the deformation of the railgun bore around the railgun joint, which is considered to be the main reason for the leakage of the plasma armature, was able to be suppressed. As a result, the compact structure of the plasma armature in the $2^{\text {nd }}$ railgun bore was generated. Based on the experimental results, we are now planning to perform the next-step experiment with higher a power supply energy.
\end{abstract}

\section{INTRODUCTION}

At the last EML conference we reported experimental results of a two-stage plasma armature railgun which was developed to achieve hypervelocities ${ }^{[1]}$. In a series of the experiments conducted to investigate the performance of the two-stage plasma armature railgun, a large increase in the projectile velocity in the $2^{\text {nd }}$ stage railgun was obtained as expected when the injection velocity into the $2^{\text {nd }}$ stage was relatively low. But, for a higher injection velocity into the $2^{\text {nd }}$ stage railgun, an unexpected physical phenomenon prevented the effective acceleration of the projectile in the $2^{\text {nd }}$ stage railgun, which was caused by the slow rise of the plasma armature velocity in the early stage of the discharge in the $2^{\text {nd }}$ stage railgun. Since the last conference we have focused our efforts on the improvement of the rise of the plasma armature velocity in the $2^{\text {nd }}$ stage railgun, but without success. Now we have another idea of a plasma armature railgun which is expected to achieve hypervelocities. The railgun has almost the same system and operational principle as those of the two-stage railgun except that two power supplies are distributed along segmented rails or continuous rails. Using continuous rails has the advantage that a plasma armature can be accelerated continuously in the whole length of the railgun by the two power supplies. But, unlike the two-stage plasma armature railgun using segmented rails, the railgun with distributed two power supplies has the problem that the discharge current from the $2^{\text {nd }}$ power supply is delivered not only to a plasma armature but also to a secondary arc. In addition to this problem, there can be another problem on the leakage of the plasma armature forward of a projectile around the railgun joint. (The whole of the railgun bore is constructed by joining two railguns and the discharge current from the $2^{\text {nd }}$ power supply is fed at the railgun joint.) If the leakage of the plasma armature is too large, it may stop the acceleration of the projectile after passing through the railgun joint. A series of experiments has been conducted to study the plasma

The Institute of Space and Astronautical Science / JAXA

3-1-1, Yoshinodai, Sagamihara City, Kanagawa 229-8510, Japan 
armature railgun with two distributed power supplies. This paper describes the major results of the experiments.

\section{SYSTEM OF THE RAILGUN WITH TWO DISTRIBUTED POWER SUUPPLIES}

Figure1 illustrates a schematic layout of the railgun with two distributed power supplies. The railgun is constructed by electrically connecting two V-block type railguns of $1 \mathrm{~m}$ and $2 \mathrm{~m}$ in each length, and is designed to feed the discharge current from the $2^{\text {nd }}$ power supply at the railgun joint. In order to reduce the effect of air drag, a rubber gasket is placed at the railgun joint to evacuate the inside of the bore of both railguns. The discharge currents from two capacitor banks $(2.5 \mathrm{mF}$ and $5 \mathrm{mF}$ ) are fed to the railgun through two pulse transformers which amplify the currents from each capacitor bank by a factor of four. The $1^{\text {st }}$ capacitor bank is turned on by receiving a trigger pulse generated manually by a master pulse generator. It is important to turn on the $2^{\text {nd }}$ capacitor bank at the right time. We use a breakwire system or a predetermined sequence system for this task. The breakwire system developed for the two-stage plasma armature railgun has a very simple circuit and is very reliable. The diagnostics to measure in-bore dynamics are as follows:

1) two Rogowski coils to measure the discharge current from the two capacitor banks;

2) eight magnetic probes to measure rail currents;

3) an X-ray system to measure the muzzle velocity

4) a voltage probe at the breech.

Figure 2 shows a cross section of the electrodes located at the railgun joint. The two electrodes are made of stainless steel and are prestressed with the torque of $250 \mathrm{~N} \cdot \mathrm{m}$. The electrodes have two functions, one is to feed the discharge current from the $2^{\text {nd }}$ power supply to the bore, and the other is to prevent the large leakage of a plasma armature in the forward direction of a projectile. The latter function in particular is considered to be a key to obtaining the successful performance of the railgun with two distributed power supplies. Some types of the electrodes have been tested in a series of the experiments. As a result, the electrodes shown in Fig. 2 have been proved most effective for preventing the leakage of the plasma armature.

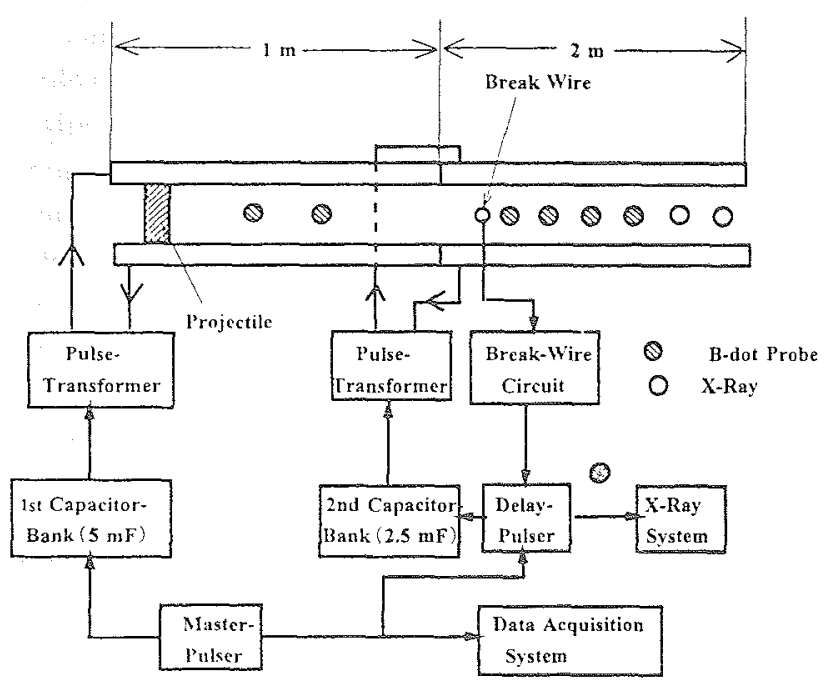

Fig. 1 Schematic layout of the railgun with two distributed power supplies

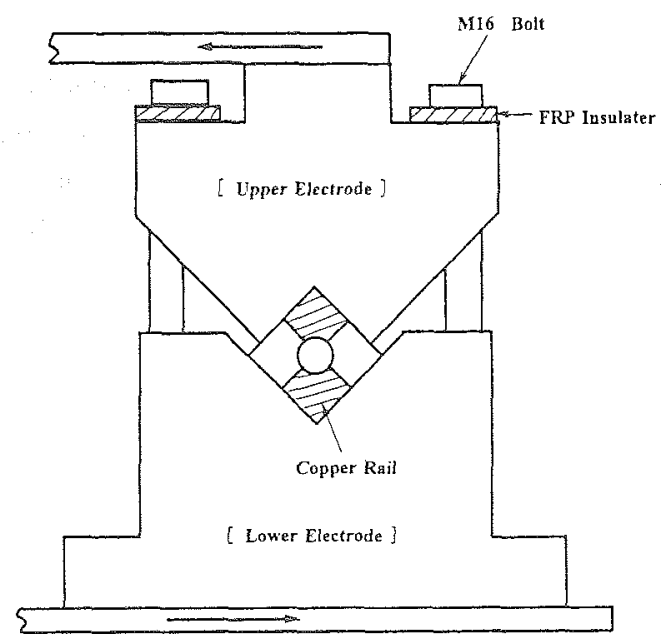

Fig. 2 Cross section of the feeding electrodes. 


\section{EXPERMENTAL RESULTS AND DISCUSSION}

A series of experiments, 11 shots, has been conducted to examine the performance of the railgun with two distributed power supplies as the first step to a hypervelocity railgun. The objectives of the experiments are as follows:

1) to confirm that a plasma armature can pass through the railgun joint without a large leakage forward of a projectile;

2) to confirm that a projectile can pass through the railgun joint without damage;

3 ) to measure the ratio of the current delivered to a plasma armature from the $2^{\text {nd }}$ power supply.

Of the 11 shots, in 8 shots the large leakage of the plasma armature forward of a projectile occurred around the railgun joint, and consequently the compact structure of the plasma armatures before entering the railgun joint disappeared within a short distance from the inlet of the $2^{\text {nd }}$ railgun. While performing these 8 shots, several types of the feeding electrodes were tested to improve the function of preventing the leakage of the plasma armature. But, the torque of bolting the electrodes were limited to only $150 \mathrm{~N} \cdot \mathrm{m}$ because the torque larger than $150 \mathrm{~N} \cdot \mathrm{m}$ caused a large deformation of the railgun bore. (The railgun bore except for the part of the joint is prestressed with the torque of about $350 \mathrm{~N} \cdot \mathrm{m}$.) As a result, the deformation of the bore due to rail currents probably leads to the occurrence of the large plasma leakage in the 8 shots. In the remaining 3 shots, the feeding electrodes shown in Fig. 2 were used. Of the 3 shots, the results of the 2 shots (Shot-I, Shot-II) are described below.

\section{[Shot-I]}

The experimental parameters are listed below.
1) $1^{\text {st }}$ capacitor bank : $99 \mathrm{~kJ}-5 \mathrm{mF} \quad 2^{\text {nd }}$ capacitor bank : $123 \mathrm{~kJ}-2.5 \mathrm{mF}$
2) projectile mass : $0.94 \mathrm{~g}$
3) muzzle velocity :5.1 km/s
4) method of triggering the discharge of the $2^{\text {nd }}$ power supply : predetermined sequence system

Figure 3 shows the configuration of B-dot probes used in this experiment. Figure 4 shows the integrated signals obtained by three B-dot probes, \#2, \#3 and \#4, and the signals of the discharge currents from the $1^{\text {st }}$ and $2^{\text {nd }}$ capacitor bank. The $2^{\text {nd }}$ capacitor bank was turned on at the time of $455 \mu$ s delayed from the start of the discharge of the $1^{\text {st }}$ power supply. The delay time of $455 \mu$ s was deduced based on previous data, and before that time the plasma armature reached around the position of the \#3 B-dot probe in front of the railgun joint as expected.

In the signal obtained by the $2 \mathrm{~B}$-dot probe, a sharp rise is seen from $370 \mu \mathrm{s}$ to $400 \mu \mathrm{s}$, indicating that the plasma armature passed through this position maintaining a compact structure. After reaching the

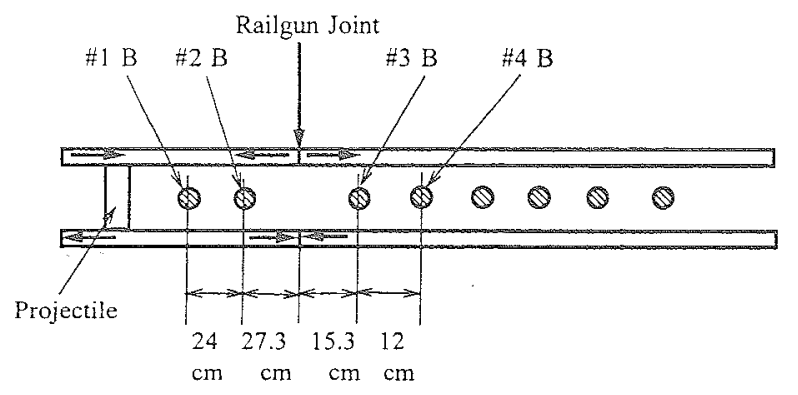

Fig. 3 Configuration of B-dot probes.

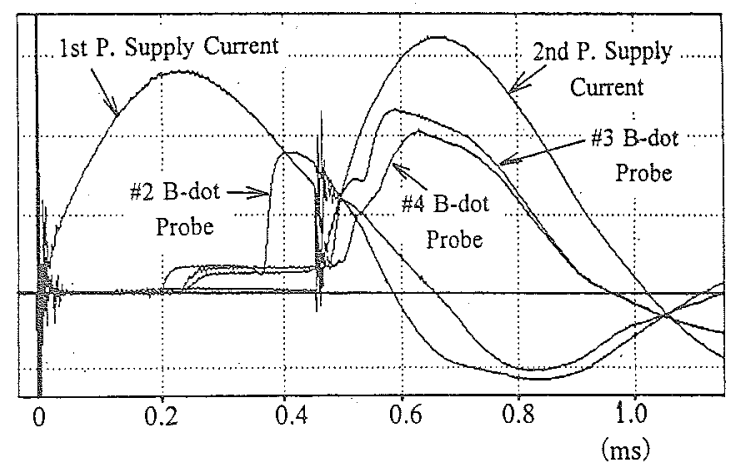

Fig. 4 Integrated signals obtained by B-dot probes and signals of the currents from two power supplies. 


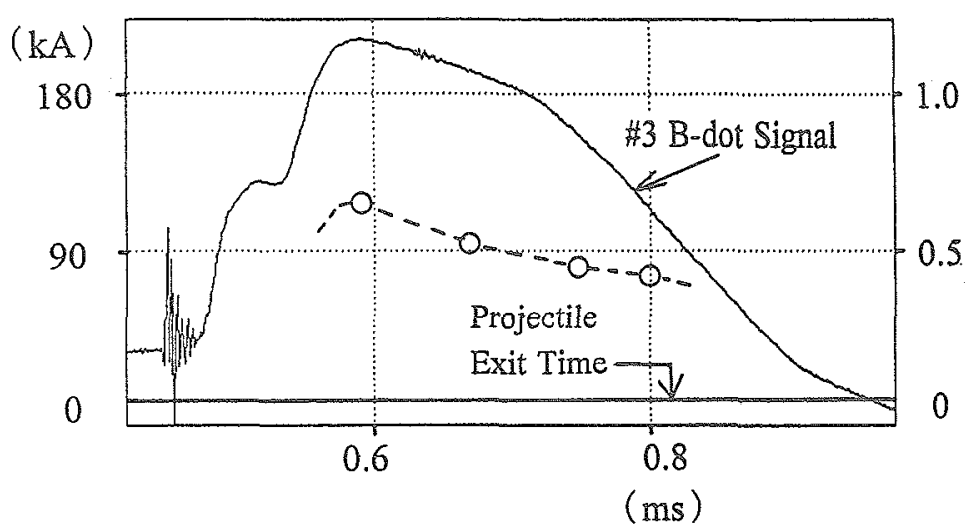

Fig. 5 Change of the rate of the current delivered to the bore region of \# 3 probe.

peak, the signal gradually decreases in the same manner the discharge current from the $1^{\text {st }}$ capacitor bank. But in the \# 3 B-dot probe signal the rising shape such as the \# 2 signal is not seen, instead it rises like the shape of a stair-case. This means that the plasma armature was separated into two lumps after passing through the position of the \# $2 \mathrm{~B}$-dot probe. The rise indicating the structure of the two separated lumps is also seen in the \# 4 B-dot probe signal. However, the slope of the rise is gentler than that of the \# 3 B-dot probe signal. Judging from these three signals, it can be presumed that the plasma armature leaked ahead of the projectile between the positions of the \# 2 and \# 3 B-dot probes, and then spread its length traveling in the bore. Actually, the signals (not shown in this paper) obtained by an array of B-dot probes installed along the bore region farther forward of the \# 4 probe position show the existence of the plasma armature further spread in the railgun bore. By visual inspection of the bore after the shot, a pit (about $2 \mathrm{~mm}$ depth, about $3 \mathrm{~mm}$ width and about $8 \mathrm{~mm}$ length) was observed along the circumference of the surface of one copper rail just at the inlet of the $2^{\text {nd }}$ railgun. The projectile was possibly damaged partly by a severe interaction between the projectile and the wall surface. The two railguns constructing the whole system were aligned with the accuracy of about $0.1 \mathrm{~mm}$ before the shot. But the deviation of the alignment of the two railguns may be caused by the repulsive force acting on the two rails by the large current. This seems to be the most probable explanation for the occurrence of the severe interaction between the projectile and the wall surface. If the leakage of the plasma armature is caused mainly by the deformation of the railgun bore around the joint area, the slope of the rise of the \# 3 signal should be gentler, which was actually seen in the 8 shots. So, it can be guessed that the leakage of the plasma armature in this shot was caused mainly by the interaction between the projectile and the bore wall, not the deformation of the railgun bore around the joint area due to a weak prestress of the feeding electrodes. Figure 5 shows the signal obtained by the \# $3 \mathrm{~B}$-dot probe and the change of the rate of the current delivered from the $2^{\text {nd }}$ capacitor bank to the bore region forward of the \# $3 \mathrm{~B}$-dot probe position. We can see that about half of the discharge current from the $2^{\text {nd }}$ capacitor bank is delivered to the bore region forward of the \# $3 \mathrm{~B}$-dot probe position, and note that the maximum values of the \# 3 and \# 4 B-dot probe signal become higher than that of the \# 2 B-dot probe. In the 8 shots with the large leakage of the plasma armature, the maximum level of the \# 3 B-dot probe signal never becomes higher than that of the \# 2 B-dot probe.

\section{[Shot-II]}

It is very difficult to control the deviation of the alignment of the two railguns so that a severe interaction between the projectile and the wall surface does not occur in the operation. So, in this shot, the inside surface of the bore was tapered with a small angle along the distance of about $10 \mathrm{~cm}$ from the inlet of the $2^{\text {nd }}$ railgun as shown in Figure 6 . This structure was chosen for the two-stage plasma armature railgun and it was already confirmed that it allowed a projectile to pass through the railgun joint without damage. 


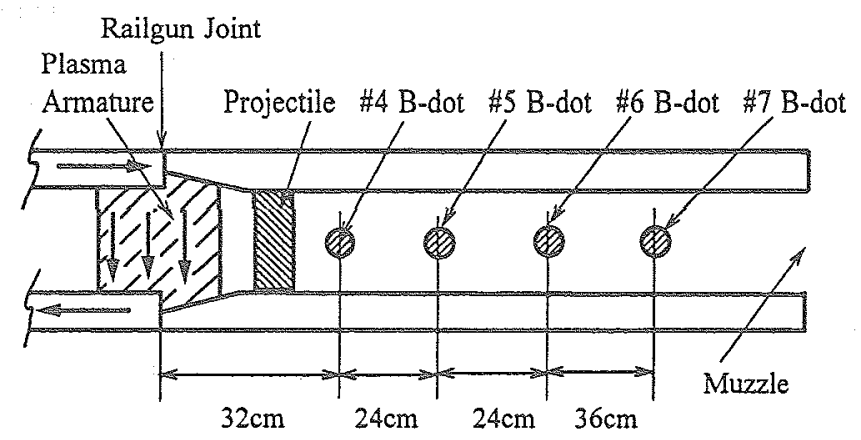

Fig. 6 Configuration of shot-II.

2nd P. Supply turns on ע

Fig. 7 Integrated signal obtained by \# 4 B-dot probe.
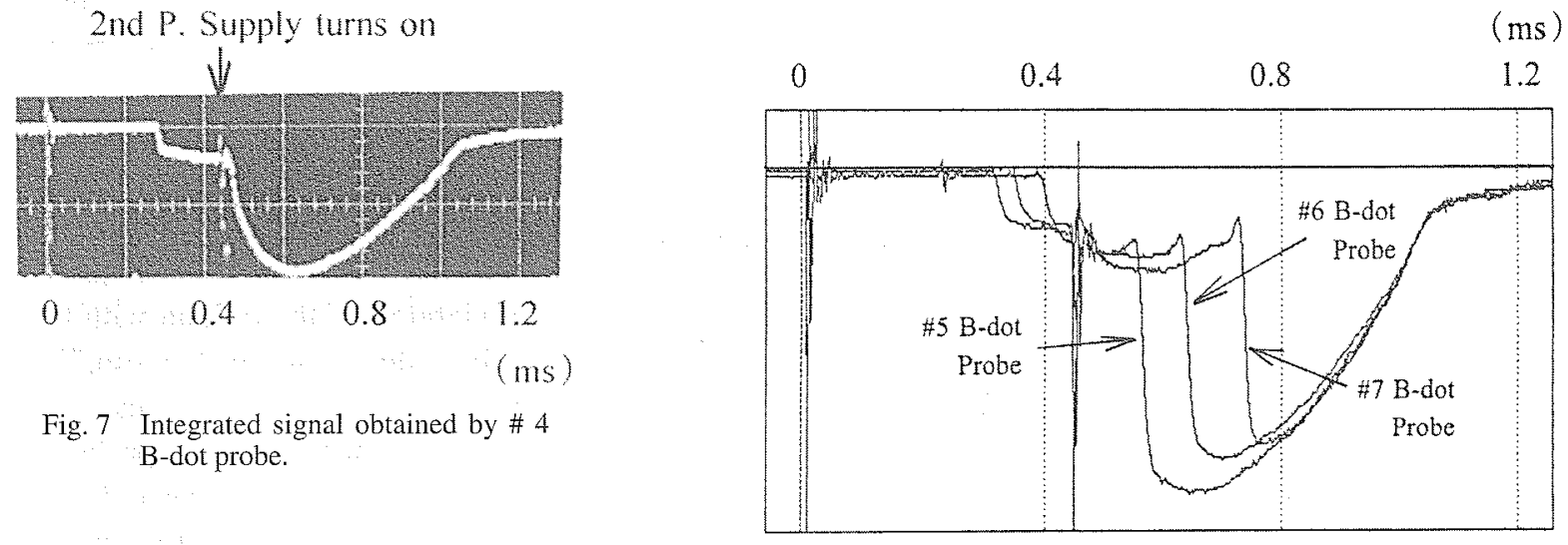

Fig. 8 Integrated signals obtained by an array of B-dot probes, from \# 5 to \# 7.

The experimental parameters are as follows;

1) $1^{\text {st }}$ capacitor bank $: 2.5 \mathrm{mF}-100 \mathrm{~kJ} \quad 2^{\text {nd }}$ capacitor bank : $5 \mathrm{mF}-100 \mathrm{~kJ}$

2 ) projectile mass : $0.83 \mathrm{~g}$

3 ) muzzle velocity : $4.1 \mathrm{~km} / \mathrm{s}$

4 ) method of triggering the $2^{\text {nd }}$ capacitor bank : breakwire system

In this shot, the breakwire system was used to turn on the $2^{\text {nd }}$ capacitor bank. The breakwire was installed instead of the B-dot probe at the position of the \# 3. Figure 7 and 8 show the integrated signals obtained by an array of the B-dot probes from \# 4 to \# 7 installed along the bore region in front of the \# 3 B-dot probe position. (These signals were recorded on two instruments, a digital memory and an oscilloscope, due to the lack of the channel number of the digital memory.) In both figures, we can see that the gentle slope of the rising wave-form in Fig. 7 is changed to the steep slope of the rising wave-form in Fig. 8. This means that the plasma armature entered the $2^{\text {nd }}$ railgun bore with an elongated structure, but without a large leakage forward of a projectile, and then the elongated structure of the plasma armature was changed to a compact structure of the plasma armature by the discharge current from the $2^{\text {nd }}$ capacitor bank while the plasma armature was traveling in the bore. As expected, the tapered structure of the inlet of the $2^{\text {nd }}$ railgun bore allowed a projectile to pass through the railgun joint without damage. But, if the plasma armature in contact with a projectile entered to the tapered region of the $2^{\text {nd }}$ railgun bore, a large leakage of the plasma armature forward of the projectile should have occurred due to the large gap between the projectile and the bore wall. However, the occurrence of the large leakage of the plasma armature was not observed.

In this shot, the discharge duration of the $1^{\text {st }}$ capacitor bank and the initial position of the projectile were 
chosen so that the discharge current decreased to around zero, but not zero, when the projectile reached at the tapered region of the $2^{\text {nd }}$ railgun. According to the data, the plasma armature has a tendency to be separated from the projectile around the end of the half cycle of the discharge current due to the decrease of the plasma armature velocity. It is presumed that the projectile and the plasma armature were separated before the projectile entered the tapered region of the $2^{\text {nd }}$ railgun bore as shown in Fig. 6, which could have led to the elongated structure of the plasma armature.

As a result, a large leakage of the plasma armature forward of the projectile was prevented. This operation system and the structure of the tapered bore of the $2^{\text {nd }}$ railgun used in this shot lead to the suppression of the large leakage of the plasma armature and give a reliable method of the projectile passing through the railgun joint. But, if a large part of the energy stored in the $2^{\text {nd }}$ capacitor bank is needed to accelerate the whole of the plasma armature until it comes in contact with the projectile, the increase in the projectile velocity by the $2^{\text {nd }}$ capacitor bank may be limited to low. In order to obtain an effective acceleration of the projectile by the $2^{\text {nd }}$ capacitor bank, the further study on the discharge duration of the $1^{\text {st }}$ capacitor bank and the structure of the tapered bore wall of the $2^{\text {nd }}$ railgun are required. Of the 3 shots, in the remaining last shot, the same operation system and configuration as the shot-II were used, and almost the same results were obtained.

\section{CONCLUSION}

A series of experiments, 11 shots, has been conducted to establish the viability of the railgun with two distributed power supplies as the first step to produce a railgun with multiple distributed power supplies. In eight shots, a deformation of the railgun bore, which occurred around the railgun joint, have caused a large leakage of the plasma armature forward of the projectile. As a result, the increase in the projectile velocity by the $2^{\text {nd }}$ capacitor bank was hardly obtained. Of remaining 3 shots, in 1 shot the projectile was damaged partly at the inlet of the $2^{\text {nd }}$ railgun bore by a severe interaction between the projectile and the bore wall. But, the large leakage of a plasma armature due to the deformation of the railgun bore was prevented by the improvement of a feeding electrode for the $2^{\text {nd }}$ capacitor bank. In this shot, it was also confirmed that about half of the discharge current from the $2^{\text {nd }}$ capacitor bank wsa delivered to a plasma armature. In the remaining 2 shots, the inside surface of the $2^{\text {nd }}$ railgun bore was tapered with a small angle along the distance of about $10 \mathrm{~cm}$ from the inlet of the bore so that a projectile passes through the railgun joint without damage. As expected, in this shot the projectile passed through the railgun joint without damage. The leakage of the plasma armature from the gap between the projectile and the tapered bore wall, in addition, was prevented by separating the projectile and the plasma armature, which was achieved by choosing the discharge duration of the $1^{\text {st }}$ capacitor bank and the initial position of a projectile. Although the three shots have been performed with a relatively low power supply energy, the three objectives for this research have been accomplished. The development directing the railgun with multiple distributed power supplies has just started. We will make our efforts focusing on the study of the generation of the compact structure of the plasma armature in the experiments performed with a higher power supply energy.

\section{REFERENCES}

[1] Akira Yamori, Migiwa Kohno, and Nobuki Kawashima, "Experimental Results of a Two-Stage Plasma Armature Railgun," IEEE TRANSACTION ON MAGNETICS, VOL. 39, NO. 1, 2003. 


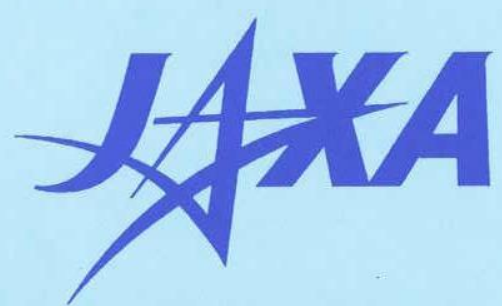

宇宙航空研究開発機構

Japan Aerospace Exploration Agency

This document is provided by JAXA. 